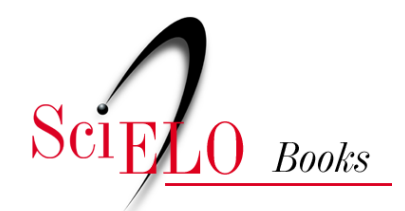

\title{
Capítulo V - Pesquisas etnobotânicas em unidades de conservação no estado do Rio de Janeiro, Brasil
}

\author{
Viviane Stern da Fonseca Kruel \\ Juan Gomes Bastos \\ Cyl Farney Catarino de Sá
}

\section{SciELO Books / SciELO Livros / SciELO Libros}

KRUEL, V.S.F., BASTOS, J.G., and SÁ, C.F.C. Pesquisas etnobotânicas em unidades de conservação no estado do Rio de Janeiro, Brasil. In: SANTOS, M.G., and QUINTERO, M., comps. Saberes tradicionais e locais: reflexões etnobiológicas [online]. Rio de Janeiro: EDUERJ, 2018, pp. 100-125. ISBN: 978-857511-485-8. https://doi.org/10.7476/9788575114858.0007.

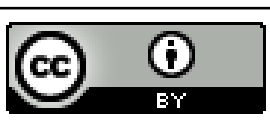

All the contents of this work, except where otherwise noted, is licensed under a Creative Commons Attribution 4.0 International license.

Todo o conteúdo deste trabalho, exceto quando houver ressalva, é publicado sob a licença Creative Commons Atribição $\underline{4.0}$.

Todo el contenido de esta obra, excepto donde se indique lo contrario, está bajo licencia de la licencia $\underline{\text { Creative }}$ Commons Reconocimento 4.0. 
$-$
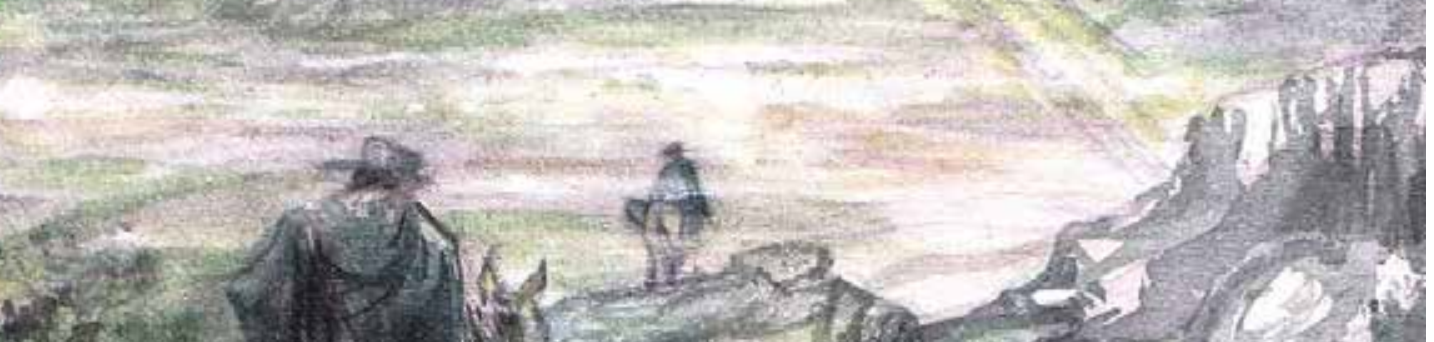

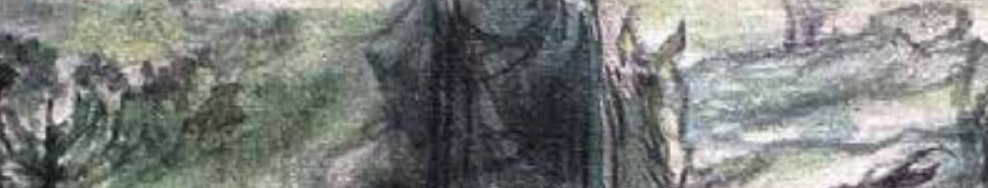
10.

A $\frac{2}{4}$ if

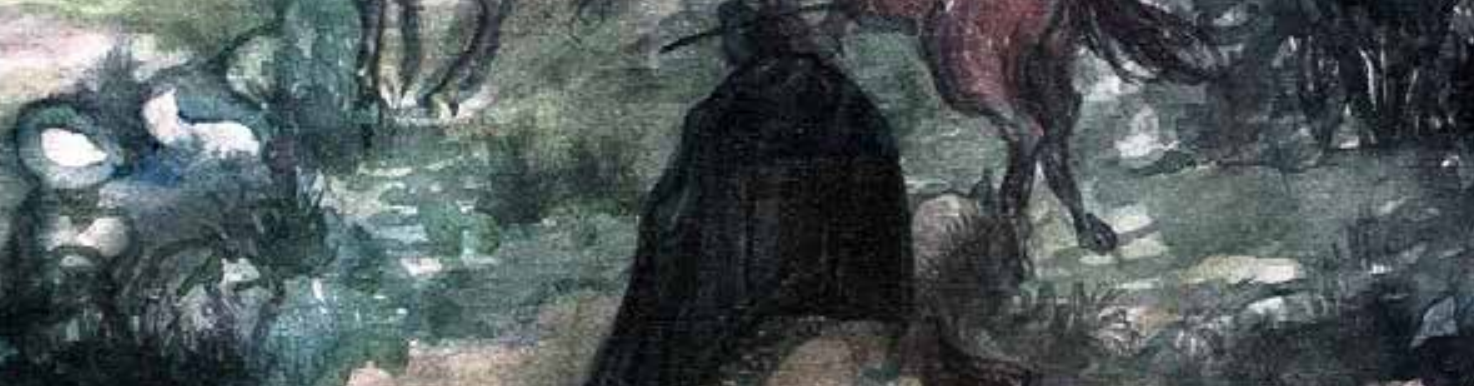
ef.

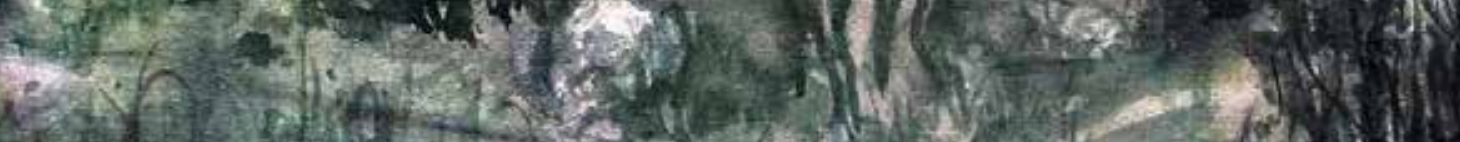

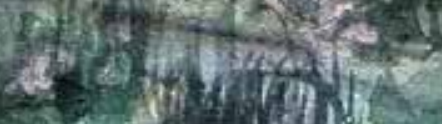
in 20 1) : 


\title{
CAPÍTULO V
}

\section{Pesquisas etnobotânicas em unidades de conservaçăo no estado do Rio de Janeiro, Brasil}

\author{
Viviane Stern da Fonseca Kruel \\ Juan Gomes Bastos \\ Cyl Farney Catarino de Sá
}

Ao longo da história das civilizações, as diferentes sociedades humanas têm buscado na natureza os recursos para a sua existência, o que tem resultado em maiores oportunidades de sobrevivência, sobretudo a partir da seleção de plantas para as mais diversas finalidades (alimentação, medicamentos, construção de moradias e embarcações etc). Da interação entre populações humanas e o uso diferenciado dos recursos vegetais, emerge a importância da disciplina etnobotânica em meio a um cenário economicamente globalizado e com tendência à perda da diversidade biológica e cultural em diversas escalas.

A etnobotânica é uma disciplina científica que estuda e interpreta a história das plantas nas sociedades antigas e atuais. ${ }^{1}$ Apresenta caráter interdisciplinar, demonstrado na diversidade de tópicos, podendo englobar tanto os fatores culturais, sociais, políticos e econômicos quanto os biológicos e ecológicos. ${ }^{2}$ Essa disciplina tem auxiliado na compreensão da interação das populações humanas com as áreas geográficas que ainda apresentam vegetação e flora nativas, assim como no fortalecimento de informações para o planejamento participativo e gestão de unidades de conservação, na troca de experiências práticas de compartilhamento nos ambientes naturais e, por conseguinte, pode ainda responder a mudanças nestes ecossistemas. ${ }^{3}$

\footnotetext{
Alexiades e Sheldon, 1996.

2 Alcorn, 1995; Cunningham, 2000; Albuquerque, 2005.

3 Berkes et al., 1995; Hanazaki, 2002.
} 
As Unidades de Conservação (UCs) - espaços criados para a conservação da biodiversidade e de processos ecológicos, no Brasil - são regulamentadas pela lei que criou o Sistema Nacional de Unidades de Conservação - SNUC, ${ }^{4}$ que "estabelece critérios e normas para a criação, implantação e gestão das unidades de conservação" (Brasil, 2000). Através dessa lei, as UCs existentes, até então, foram distribuídas em doze categorias, sendo cinco delas de proteção integral (Parque Nacional, Reserva Biológica, Estação Ecológica, Monumento Natural e Refúgio de Vida Silvestre) e sete de uso sustentável (Floresta Nacional, Área de Proteção Ambiental, Área de Relevante Interesse Ecológico, Reserva Extrativista, Reserva de Fauna, Reserva de Desenvolvimento Sustentável, Reserva Particular do Patrimônio Natural). Apesar dessa organização, Dourojeanni e Pádua (2013) tecem algumas interessantes críticas e reflexões sobre o excesso de categorias de UCs no Brasil, além do fato de que poderiam ser reduzidas pela metade, dado o nível de confusão gerado por diferenças muito sutis entre categorias.

A "Lei do SNUC", segundo Santilli (2014), além de definir as doze categorias de UCs, ressalta, dentre os principais objetivos: "proteger os recursos naturais à subsistência de populações tradicionais, respeitando e valorizando seu conhecimento e sua cultura e promovendo-as social e economicamente". A autora destaca ainda que "o conjunto de políticas públicas reforça a necessidade de uma interpretação sistêmica e integrada dos instrumentos legais de proteção ao meio ambiente e cultura".

As primeiras UCs criadas no Brasil foram os Parques Nacionais, cujos fundamentos para a criação, de acordo com Barros (1952), seriam "a topografia, a geologia, os ambientes floro-faunianos, os acidentes criados pela natureza no seu longo processo evolutivo". Rylands \& Brandon (2005), ao analisarem as UCs brasileiras criadas até aquele momento, relatam que, além dos critérios biogeográficos aplicados entre 1980-90, outros três critérios mais recentes: os corredores de biodiversidade, os workshops para a definição de áreas prioritárias e o programa de áreas protegidas para a Amazônia (ARPA). A primeira UC brasileira (um parque nacional) foi criada no estado do Rio de Janeiro, em 1937, a partir da Estação Biológica do Jardim Botânico do Rio de Janeiro - o Parque Nacional do Itatiaia. Esse parque, situado numa das montanhas mais altas do país, está entre

\footnotetext{
4 Lei no 9.985 de 18 de julho de 2000.
} 
os principais parques nacionais do Brasil ${ }^{5}$ e é detentor de uma significativa riqueza de espécies de Angiospermas, estimada atualmente em 1.206 táxons e com muitas espécies endêmicas. ${ }^{6}$ Ele continua a ser ativamente estudado e, de acordo com Bittencourt e Paula (2012), guardadas as limitações metodológicas, foi dentre os parques nacionais brasileiros o que registrou o maior número de publicações científicas até 2012. Merecem também destaque as mais de 9.000 amostras de plantas depositadas no Herbário do Jardim Botânico do Rio de Janeiro (RB) e que espelham também a atividade científica em botânica nesse parque nacional.

O exemplo acima demonstra que a riqueza de espécies desta e de muitas outras UCs também necessita ser documentada e relacionada à história de uso da flora e da vegetação nativa por comunidades tradicionais que habitam o seu entorno e que ainda detém o conhecimento sobre espécies, usos e suas áreas de ocorrência. Dessa forma, o presente estudo visou traçar um panorama das pesquisas etnobotânicas realizadas nas UCs e seus entornos situadas no estado do Rio de Janeiro, a partir de consultas à artigos e bases de dados de teses e dissertações nos portais: CAPES, Domínio Público, Scielo e Web of Science. Foram considerados os estudos com ênfase em indicações de plantas úteis relacionadas à vegetação das UCs ou em suas proximidades, entretanto os estudos de mercado, feiras livres, comercialização de plantas não foram aqui analisados. De maneira complementar, foi consultado o Cadastro Nacional de Unidades de Conservação - Ministério do Meio Ambiente (MMA) e as Unidades de Conservação do Instituto Estadual do Ambiente (INEA). ${ }^{8}$

\section{As unidades de conservaçầo fluminenses}

O papel estratégico no estabelecimento e manejo de áreas protegidas e de UCs vem se tornando cada vez mais evidente e urgente no Brasil, em função do crescimento e expansão da população sobre áreas que não deveriam ser ocupadas como estão hoje às margens de rios e lagoas, estu-

\footnotetext{
ICMBio, 2018.

6 Baumgratz et al., 2014.

MMA, 2018.

8 INEA, 2018a.
} 
ários, encostas íngremes e áreas de restingas. Invariavelmente, as tragédias ocorridas no passado recente do país (região serrana fluminense, em 2011, inundações no norte do país e escassez de água para abastecimento no sudeste) têm sido atribuídas unicamente à natureza, o que de certa forma minimiza o papel das ações humanas.

O "Cadastro Nacional de Unidades de Conservação"9 contém 684 UCs de Proteção Integral, sendo 367 estaduais (159.190ha), e 1.462 UCs de Uso Sustentável (538 estaduais em 607.626ha). De acordo com esse cadastro, só no estado do Rio de Janeiro foram contabilizadas 295 UCs (112 estaduais, 84 federais e 99 municipais), sendo 79 de proteção integral (tabela 1), com destaque para os parques naturais municipais $(48,1 \%)$ e os parques estaduais (13,9\%). Dentre as 216 UCs de Uso Sustentável fluminense (tabela 2), destacam-se as Reservas Particulares do Patrimônio Natural, estaduais e federais (68,5\%), e as Áreas de Proteção Ambiental - APAs (28,7\%).

Tabela 1. As Unidades de Conservaçăo de Proteçăo Integral no estado do Rio de Janeiro

\begin{tabular}{|l|c|c|c|c|}
\hline Categorias & Federal & Estadual & Municipal & Total \\
\hline Estação Ecológica & 2 & 1 & 0 & 3 \\
\hline Reserva Biológica & 3 & 3 & 1 & 7 \\
\hline Parque & 5 & 11 & 38 & 54 \\
\hline Monumento Natural & 1 & 0 & 8 & 9 \\
\hline Refúgio da Vida Silvestre & 0 & 0 & 6 & 6 \\
\hline Total & 11 & 15 & 53 & 79 \\
\hline
\end{tabular}

Fonte: MMA 2018 - Cadastro Nacional de Unidades de Conservaçăo.

As categorias de UC de Proteção Integral são responsáveis por cerca de $46,7 \%$ do total da superfície de áreas protegidas no Brasil, principalmente pelos parques que representam $61,4 \%$ desse total. ${ }^{10}$ No estado do Rio de Janeiro (tabela 1), não é muito diferente. Os parques representam

\footnotetext{
${ }^{9} \mathrm{MMA}, 2018$.

${ }^{10} \mathrm{MMA}, 2018$.
} 
68,3\% das UCs de Proteção Integral e os cinco parques nacionais, situados no estado, detêm $170.806,98$ ha de áreas protegidas, enquanto outros 184.226ha são protegidos em onze parques estaduais ( $41 \%$ das áreas protegidas estaduais). As categorias de proteção integral são aquelas onde a proteção da biodiversidade se dá de forma mais efetiva devido as limitações de uso descritas na Lei do SNUC.

Tabela 2. As Unidades de Conservaçầo de Uso Sustentável no estado do Rio de Janeiro

\begin{tabular}{|l|c|c|c|c|}
\hline Categorias & Federal & Estadual & Municipal & Total \\
\hline $\begin{array}{l}\text { Área de Proteção Ambiental } \\
\text { (APA) }\end{array}$ & 5 & 13 & 44 & 62 \\
\hline $\begin{array}{l}\text { Área de Relevante Interesse } \\
\text { Ecológico (ARIE) }\end{array}$ & 1 & 0 & 2 & 3 \\
\hline Floresta Nacional (FLONA) & 1 & 0 & 0 & 1 \\
\hline Reserva Extrativista (RESEX) & 1 & 0 & 0 & 1 \\
\hline Reserva de Fauna & 0 & 0 & 0 & 0 \\
\hline $\begin{array}{l}\text { Reserva de Desenvolvimento } \\
\text { Sustentável (RDS) }\end{array}$ & 0 & 1 & 0 & 1 \\
\hline $\begin{array}{l}\text { Reserva Particular do Patri- } \\
\text { mônio Natural (RPPN) }\end{array}$ & 65 & 83 & 0 & 148 \\
\hline Total & 73 & 97 & 46 & 216 \\
\hline
\end{tabular}

Fonte: MMA 2018 - Cadastro Nacional de Unidades de Conservaçăo.

Dentre as UCs de Uso Sustentável, as RPPNs merecem um destaque especial em termos de análise, já que, de acordo com o SNUC, as RPPNs têm como objetivo a conservação da diversidade biológica, sendo somente admitidas a pesquisa científica e a visitação com objetivos turísticos, recreativos e educacionais. Nesse aspecto, Dourejanni e Pádua (2013), no texto Para melhor desmantelar o paraiso, esclarecem que na verdade essa categoria de UC é de fato e de direito uma UC de Proteção Integral. Assim sendo, o Estado de Santa Catarina, através da Lei n. 11.986 de 12 de novembro de 
2001, já havia enquadrado as RPPNs como UC de Proteção Integral. Atualmente, existem 859 RPPNs cadastradas no Brasil (MMA, 2018). Embora em escalas reduzidas, essas áreas representam 561.900 ha de áreas protegidas, ou seja, menos de $1 \%$ do total de UCs de Uso Sustentável cadastradas.

O "sucesso" inicial da RPPN pode ser atribuído ao fato de ela poder ser criada voluntariamente pelo proprietário das terras, através da submissão de reconhecimento por ato do poder público. Entretanto, Dourejanni e Pádua (2013), baseados em dados do Ibama entre 1990 e 2002, indicaram, no texto Vitimando as reservas particulares, que 440.000 ha de RPPNs já haviam sido criadas. Segundo os autores, esse número recrudesceu desde então devido ao excesso de burocracia no Decreto n. ${ }^{\circ} 4.340$ de 2002, à necessidade de apresentar um plano de manejo e, de acordo com a Instrução Normativa n. 24 de 2004, a um roteiro metodológico exaustivo e extremamente técnico e bastante oneroso para quem decida criar uma RPPN.

As RPPNs vêm sendo uma das categorias de UCs mais peculiares no ordenamento jurídico brasileiro, devido às suas características de domínio e gestão privada da unidade e a participação direta do particular na criação da UC, dando efetividade ao princípio da participação da sociedade na tutela do meio ambiente. No Estado do Rio de Janeiro, existem 148 RPPNs, sendo 83 de âmbito estadual e reconhecidas pelo INEA, e somam 7.154,01ha. ${ }^{11}$ Os municípios de Nova Friburgo, Silva Jardim e Varre-Sai possuem a maior representatividade em relação ao número de RPPNs estaduais por município (figura 1). No âmbito federal, há 65 RPPNs, totalizando 5.925,93ha de área, ${ }^{12}$ sendo os municípios de Silva Jardim, Nova Friburgo e Varre-sai aqueles com maior número de RPPNs por município (figura 1). Até o momento, a maior RPPN no estado do RJ é a Fazenda Caruara, de âmbito estadual (INEA/RJ/PRES n. 357 de 19 de julho de 2012), localizada em São João da Barra (3.844,73ha), seguida por: Fazenda Cachoeirinha - âmbito federal, em Mangaratiba (650ha); Santo Antônio - âmbito estadual, em Resende (538,59ha); Fazenda Santa Izabel - âmbito federal, em Mangaratiba (525ha); Três Morros - âmbito federal, em Casimiro de Abreu (508,78ha). No município de Silva Jardim, encontram-se grandes áreas de RPPN, de âmbito federal, como: a Fazenda Bom Retiro (472ha), Floresta Alta (380,9ha) e União $\left(343,1\right.$ ha) ${ }^{13}$

\footnotetext{
${ }^{11}$ INEA, 2018b.

${ }^{12}$ MMA - Cadastro Nacional de Unidades de Conservação, 2018.

${ }^{13}$ MMA - Cadastro Nacional de Unidades de Conservação, 2018.
} 
Figura 1. Os municípios do estado do Rio de Janeiro com até cinco RPPNs reconhecidas pelo ICMBio e INEA

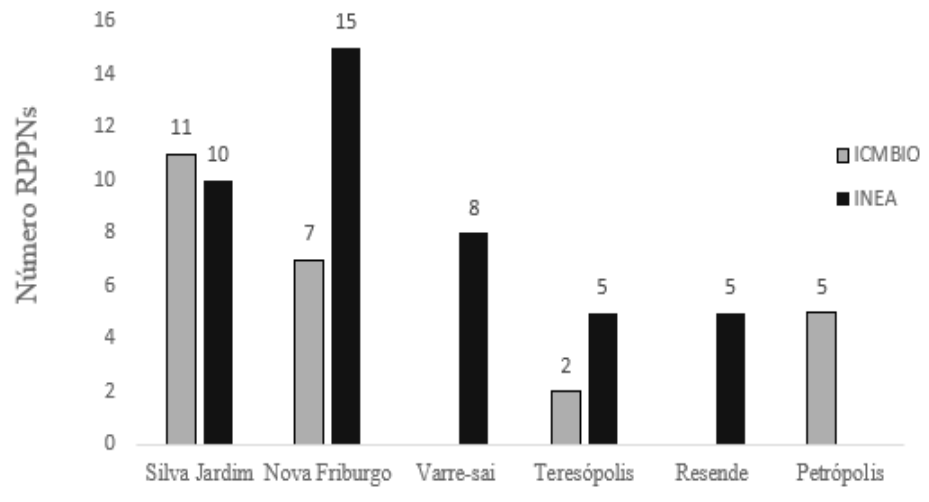

Municípios

Fonte: MMA, 2018 e INEA, 2018.

As APAs estaduais e federais (tabela 2) constituem a segunda categoria de UC de Uso Sustentável mais abundante no estado do Rio de Janeiro. Essas APAs têm uma abrangência diversificada em relação aos municípios e tipos de ambientes (costeiros, como as restingas, quanto para o bioma mata atlântica strictu sensu). Dentre todas as categorias de UCs, as APAs são as de pior reputação, ${ }^{14}$ já que muitas delas não cumprem seu papel para o qual foi criado, muito pelo fato de terem sido criadas sobre terras privadas, ou pretensamente privadas, e que estão a todo tempo ao sabor de pressões políticas e de zoneamentos viciados e de planos de manejo fantasiosos. Nesse aspecto, as APAs estaduais da Região dos Lagos (Maricá, Pau-Brasil, Serra da Sapiatiba e Massambaba) são exemplos claros de áreas que servem muito mais à especulação imobiliária do que à conservação da biodiversidade.

${ }^{14}$ Dourejanni e Pádua, 2013. 


\section{Panorama das pesquisas etnobotânicas}

Nesta análise, consideramos 41 estudos etnobotânicos realizados em UCs e em suas proximidades (tabelas 3 e 4 ). Desse total, $78 \%$ foram referentes a estudos publicados em periódicos e livros e $22 \%$ a dissertações e teses não publicadas (figura 3). Nas UCs de Proteção Integral encontramos 47\% dos trabalhos realizados em parques estaduais e proximidades (figura 3), seguido pelos parques nacionais (23,5\%) e reservas biológicas (17,6\%). Para as UCs de Uso Sustentável, nove estudos foram relacionados, ${ }^{15}$ sendo seis destes em Área de Proteção Ambiental (tabelas 3 e 4).

A maior parte dos estudos foi realizada em áreas de Floresta Atlântica s.l. (principalmente Floresta Ombrófila Densa Montana e Submontana), seguidos por áreas de restinga, embora existam outras formações associadas, como os campos de altitude, que estão exclusivamente em UCS de Proteção Integral.

Dentre os grupos humanos, residentes no entorno das UCs, relacionados nos artigos desta análise, identificamos principalmente: os sitiantes e comunidades rurais (56\%), os pescadores artesanais e caiçaras $(26,8 \%)$, e as comunidades quilombolas $(4,8 \%)$. Vale destacar que $17 \%$ dos artigos não indicaram os grupos humanos abordados (tabelas 3 e 4). Ressalta-se ainda que o esforço amostral, bem como o enfoque e o número de espécies relacionadas como úteis foram diferentes entre os trabalhos, tendo predominado a categoria de uso medicinal, com 30\% das publicações (tabelas 3 e 4).

Em relação à riqueza de espécies identificadas nos trabalhos, salienta-se que treze deles indicaram mais de cem espécies como úteis para as proximidades das UCs de Proteção Integral (tabela 3), enquanto que, para as UCs de Uso Sustentável (tabela 4), a riqueza de espécies só ultrapassou oitenta citadas como úteis, quando o trabalho também se relacionava com UCs de Proteção Integral (ver nas tabelas 3 e 4).

15 Figueiredo et al., 1997; Fonseca-Kruel e Peixoto, 2004; Borges e Peixoto, 2009; Patzlaff et al., 2011; Boscolo e Fernandes, 2014; Baldini, 2015; Quintero et al., 2015; Mourão et al., 2017; Valverde et al., 2018. 
Figura 2. Análise das UCs de Proteçầo Integral e de Uso Sustentável em relaçăo ao desenvolvimento de pesquisas etnobotânicas no estado do Rio de Janeiro

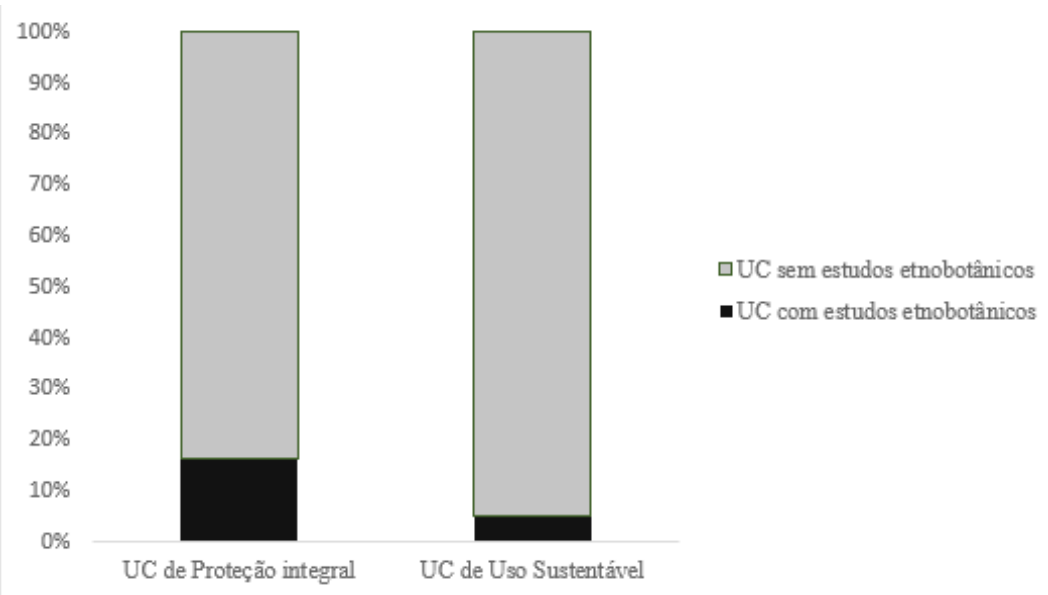

Figura 3. Análise das UCs em relaçăo à presença de dissertaçôes/teses de pós-graduaçăo ou artigos publicados

30
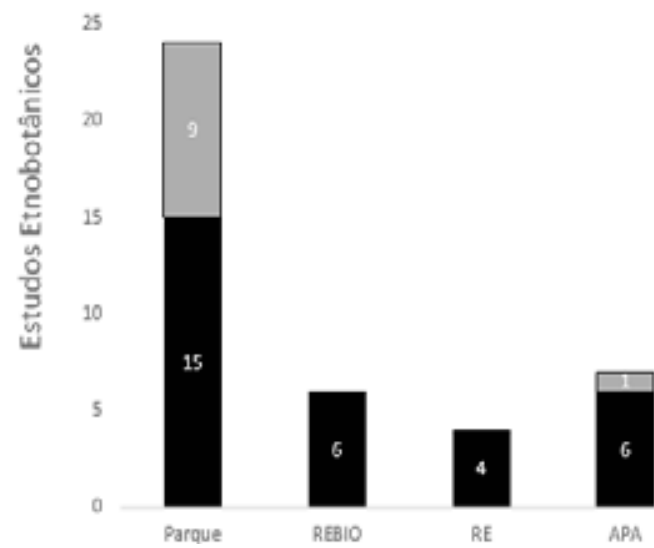

口Dissertaçles ou Teses

-Artigos

REBIO

RE

APA

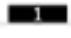

RESEX

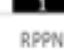




\section{Áreas prioritárias para pesquisas etnobotânicas}

A partir das análises das UCs situadas no estado do Rio de Janeiro (tabelas 1 e 2) e dos trabalhos ali desenvolvidos, na linha de etnobotânica (tabela 3 e 4; figura 3), verificamos que existe um potencial para pesquisas nas UCs de Uso Sustentável (tabela 4; figura 2 e 3). Isto se dá pelo fato de que $73,2 \%$ das UCs fluminenses estão na categoria de uso sustentável, e apenas 3\% dos trabalhos analisados foram ali realizados. Assim, as categorias APA e RPPN possibilitam maiores oportunidades e potencialidades para o desenvolvimento das comunidades humanas locais, tanto pelo número de UCs dessas categorias quanto pelas extensões de áreas protegidas e ambientes diferentes.

Outro fato a salientar é que de acordo com o SNUC, as RPPNs permitem visitação com objetivos turísticos, recreativos e educacionais, enquanto que as APAs, além da permissão de visitação, admitem o uso dos recursos naturais de maneira sustentável. A realização dessas atividades pela população local pode gerar oportunidades de desenvolvimento local para a complementação da geração de renda e emprego, resultando no aumento da qualidade de vida sem prejuízo à conservação ambiental.

Dos nove trabalhos realizados nas UCs de Uso Sustentável (tabela 4), três foram realizados no litoral e com enfoque no saber sobre o uso de plantas por pescadores artesanais e/ou caiçaras. Pelo menos em duas dessas áreas já existiam estudos sobre a vegetação e a flora local, como a APA Cairuçu e a RESEX de Arraial do Cabo (área limítrofe ao Parque Estadual da Costa do Sol), o que respalda a pesquisa sobre a flora útil local. Além dos artigos publicados, outras ferramentas que não devem ser negligenciadas na escolha de áreas para desenvolvimento de novos projetos são as consultas aos herbários virtuais e a Lista de Espécies da Flora do Brasil. No caso do estado do Rio de Janeiro, essa ferramenta é fundamental, pois, das 216 UCs de Uso Sustentável distribuídas pelo estado, 24\% são RPPNs concentradas em três municípios da Região Serrana (figura 1). Essa região é também uma das mais bem conhecidas em relação à sua riqueza florística (citada em diversos artigos científicos) e à densidade de coletas botânicas, de acordo com a consulta ao Banco de Dados JABOT do Jardim Botânico do Rio de Janeiro. 
Muito embora as UCs de Proteção Integral tenham o objetivo básico, de acordo com o SNUC, de "[...] preservar a natureza, sendo admitida apenas o uso indireto dos seus recursos naturais $[. .$.$] ", constatamos que$ em $16,4 \%$ delas (tabelas 1, 3 e 4) foram desenvolvidas $82,9 \%$ das pesquisas etnobotânicas. Esse dado revela ainda (tabela 3) que trinta dos trabalhos foram realizados após o ano 2000, ou seja, são estudos recentes em UCs criadas recentemente, enquanto no Parque Nacional de Itatiaia e na Rebio Poço das Antas, as mais antigas dentre as UCs estudadas (tabela 3), foram realizadas $20,5 \%$ das pesquisas. Tal fato demonstra que nas UCs de Proteção Integral há possibilidades de desenvolvimento de pesquisas, mas com limitação de ações e aplicações, já que não podem estar em conflito com as premissas desta categoria conforme o SNUC. Outro fato importante a destacar, no que concordamos com a observação de Luz e Elias (2014), é a necessidade de consulta ao Plano de Manejo (se existir!) da UC a ser pesquisada, de forma que seja verificada ali as prioridades de pesquisa da UC. Essa forma de agir torna o pesquisador um parceiro e não um simples usuário da UC, na medida em que sua pesquisa, quando possível, auxilia na gestão, proteção e conservação da área.

As questões socioeconômicas estão diretamente relacionadas às UCs, sendo os principais tópicos: a pressão demográfica no entorno dos limites do parque; as atitudes da população local, no entorno e dentro da UC; a economia local e a dependência de atividades extrativas. Nesse aspecto, a etnobotânica tem um papel fundamental para promover a conciliação do desenvolvimento humano, sobrevivência cultural e a conservação dos recursos vegetais em vários níveis - variedades, espécies, populações (Minnis, 2000; Tuxill e Nabhan, 2001). 
Tabela 3. As UCs de Proteçăo Integral (federais e estaduais) localizadas no estado do Rio de Janeiro com publicaçồes em etnobotânica. Legenda: PN (Parque nacional) - PNI (PN do Itatiaia), PNRJ (PN da Restinga de Jurubatiba), PNSO (PN Serra dos Orgăos). PE (Parque estadual) - PEC (PE do Cunhambebe), PECS (PE da Costa do Sol), PEPB (PE da Pedra Branca), PEPS (PE da Pedra Selada), PESET (PE da Serra da Tiririca), PETP (PE dos Três Picos), PEIG (PE da llha Grande), REJ (Reserva Ecológica da Juatinga), REBI0 (Reserva Biológica). (a) Populaçăo tradicional, (b) Populaçăo năo tradicional, (s/i) Sem informaçầo disponivel e/ou definida, (*) Pesquisa relevante em mais de uma UC.

\begin{tabular}{|c|c|c|c|c|c|}
\hline UC & Gestão & $\begin{array}{l}\text { Autor/Tipo de Publi- } \\
\text { cação }\end{array}$ & Grupos estudados & Resultados & Obtenção dos dados \\
\hline \multirow{4}{*}{ PNI } & \multirow{4}{*}{ ICMBIO } & $\begin{array}{l}\text { Magnanini (2005) } \\
\text { Dissertação ou tese }\end{array}$ & $\begin{array}{l}\text { (a) sitiantes, comunidade } \\
\text { rural } \\
\text { (b) funcionários da UC }\end{array}$ & $\begin{array}{c}81 \text { espécies; } \\
1 \text { categoria de uso }\end{array}$ & Entrevistas semiestruturadas \\
\hline & & $\begin{array}{c}\text { Baldini e Silva (2007) } \\
\text { Artigo }\end{array}$ & $\begin{array}{c}\text { (a) sitiantes, comunidade } \\
\text { rural }\end{array}$ & $\begin{array}{c}49 \text { espécies; } \\
9 \text { categorias de uso }\end{array}$ & Entrevistas semiestruturadas \\
\hline & & $\begin{array}{c}\text { Baldini (2008) } \\
\text { Dissertação ou tese }\end{array}$ & $\begin{array}{c}\text { (a) sitiantes, comunidade } \\
\text { rural }\end{array}$ & $\mathrm{s} / \mathrm{i}$ & $\mathrm{s} / \mathrm{i}$ \\
\hline & & $\begin{array}{l}\text { Quinteiro et al. (2015) } \\
\text { Artigo }\end{array}$ & (a) sitiantes & $\begin{array}{c}198 \text { espécies; } \\
8 \text { categorias de uso }\end{array}$ & Entrevistas semiestruturadas \\
\hline PNRJ & ICMBIO & $\begin{array}{l}\text { Santos et al. (2009) } \\
\text { Artigo }\end{array}$ & $\begin{array}{l}\text { (a) pescadores artesanais, } \\
\text { caçadores }\end{array}$ & $\begin{array}{c}119 \text { espécies; } \\
8 \text { categorias de uso }\end{array}$ & Entrevistas semiestruturadas \\
\hline
\end{tabular}




\begin{tabular}{|c|c|c|c|c|c|}
\hline UC & Gestão & $\begin{array}{l}\text { Autor/Tipo de Publi- } \\
\text { cação }\end{array}$ & Grupos estudados & Resultados & Obtenção dos dados \\
\hline \multirow{2}{*}{ PNRJ } & \multirow{2}{*}{ ICMBIO } & $\begin{array}{c}\text { Boscolo e Valle (2008) } \\
\text { Artigo }\end{array}$ & $\mathrm{s} / \mathrm{i}$ & $\begin{array}{c}91 \text { espécies; } \\
1 \text { categoria de uso }\end{array}$ & Entrevistas semiestruturadas \\
\hline & & $\begin{array}{l}\text { Maioli-Azevedo e Valle } \\
\qquad \begin{array}{c}\text { (2011) } \\
\text { Artigo }\end{array}\end{array}$ & (a) quilombolas & $\begin{array}{c}220 \text { espécies; } \\
9 \text { categorias de uso }\end{array}$ & Entrevistas semiestruturadas \\
\hline PNSO & ICMBIO & $\begin{array}{c}\text { Souza (2014) } \\
\text { Dissertação ou tese }\end{array}$ & (a) sitiantes & 1 categoria de uso & Entrevistas semiestruturadas \\
\hline \multirow{2}{*}{ PEC } & \multirow{2}{*}{ INEA } & $\begin{array}{c}\text { Medeiros et al. (2004) } \\
\text { Artigo }\end{array}$ & (a) sitiantes & $\begin{array}{c}36 \text { espécies; } \\
1 \text { categoria de uso }\end{array}$ & Entrevistas semiestruturadas \\
\hline & & $\begin{array}{l}\text { Medeiros et al. (2005) } \\
\text { Artigo }\end{array}$ & (a) sitiantes & $\begin{array}{c}34 \text { espécies; } \\
1 \text { categoria de uso }\end{array}$ & Entrevistas semiestruturadas \\
\hline \multirow{2}{*}{ PECS } & \multirow{2}{*}{ INEA } & $\begin{array}{l}\text { Fonseca-Kruel et al. } \\
\qquad \begin{array}{c}\text { (2009) } \\
\text { Artigo }\end{array}\end{array}$ & (a) pescadores artesanais & $\begin{array}{c}41 \text { espécies; } \\
5 \text { categorias de uso }\end{array}$ & Entrevistas semiestruturadas \\
\hline & & $\begin{array}{l}\text { Fonseca-Kruel (2011) } \\
\text { Dissertação ou tese }\end{array}$ & $\begin{array}{l}\text { (a) pescadores artesanais, } \\
\text { quilombolas, sitiantes }\end{array}$ & $\begin{array}{c}204 \text { espécies; } \\
7 \text { categorias de uso }\end{array}$ & Entrevistas semiestruturadas \\
\hline
\end{tabular}




\begin{tabular}{|c|c|c|c|c|c|}
\hline UC & Gestão & $\begin{array}{l}\text { Autor/Tipo de Publi- } \\
\text { cação }\end{array}$ & Grupos estudados & Resultados & Obtenção dos dados \\
\hline PECS & INEA & $\begin{array}{c}\text { van Luijk e Fonseca- } \\
\text {-Kruel (2017) } \\
\text { Dissertação ou tese }\end{array}$ & $\begin{array}{l}\text { (b) alunos do ensino fun- } \\
\text { damental }\end{array}$ & $\begin{array}{c}25 \text { espécies; } \\
4 \text { categorias de uso }\end{array}$ & Entrevistas semiestruturadas \\
\hline PEPB & INEA & $\begin{array}{l}\text { Magalhães (2010) } \\
\text { Dissertação ou tese }\end{array}$ & (a) sitiantes & $\begin{array}{c}221 \text { espécies; } \\
8 \text { categorias de uso }\end{array}$ & Entrevistas semiestruturadas \\
\hline PEPS & INEA & $\begin{array}{c}{ }^{\star} \text { Baldini (2015) } \\
\text { Dissertação ou tese }\end{array}$ & sitiantes, comunidade rural & $\begin{array}{c}294 \text { espécies: } \\
6 \text { categorias de uso }\end{array}$ & Entrevistas semiestruturadas \\
\hline \multirow{4}{*}{ PESET } & \multirow{4}{*}{ INEA } & $\begin{array}{c}\text { Lopes e Andreata } \\
\text { (1989) } \\
\text { Artigo }\end{array}$ & $\mathrm{s} / \mathrm{i}$ & $\begin{array}{c}7 \text { espécies; } \\
1 \text { categoria de uso }\end{array}$ & $\mathrm{s} / \mathrm{i}$ \\
\hline & & $\begin{array}{l}\text { Lopes e Andreata } \\
\qquad(1990) \\
\text { Artigo } \\
\end{array}$ & $\mathrm{s} / \mathrm{i}$ & $\begin{array}{c}6 \text { espécies; } \\
1 \text { categoria de uso }\end{array}$ & $\mathrm{s} / \mathrm{i}$ \\
\hline & & $\begin{array}{l}\text { Lopes e Andreata } \\
\qquad \begin{array}{c}(1991) \\
\text { Artigo }\end{array}\end{array}$ & $\mathrm{s} / \mathrm{i}$ & $\begin{array}{c}11 \text { espécies; } \\
1 \text { categoria de uso }\end{array}$ & $\mathrm{s} / \mathrm{i}$ \\
\hline & & $\begin{array}{l}\text { Lopes e Andreata } \\
\qquad \begin{array}{c}(1992) \\
\text { Artigo }\end{array}\end{array}$ & $\mathrm{s} / \mathrm{i}$ & $\begin{array}{l}10 \text { espécies; } \\
1 \text { categoria de uso }\end{array}$ & $\mathrm{s} / \mathrm{i}$ \\
\hline
\end{tabular}




\begin{tabular}{|c|c|c|c|c|c|}
\hline UC & Gestão & $\begin{array}{l}\text { Autor/Tipo de Publi- } \\
\text { cação }\end{array}$ & Grupos estudados & Resultados & Obtenção dos dados \\
\hline PESET & INEA & $\begin{array}{c}\text { Lopes et al. (2004) } \\
\text { Artigo }\end{array}$ & $\mathrm{s} / \mathrm{i}$ & $\begin{array}{c}56 \text { espécies; } \\
1 \text { categoria de uso }\end{array}$ & $\mathrm{s} / \mathrm{i}$ \\
\hline PETP & INEA & $\begin{array}{c}\text { Boscolo (2011) } \\
\text { Dissertação ou tese }\end{array}$ & (a) sitiantes & $\begin{array}{c}369 \text { espécies; } \\
4 \text { categorias de uso }\end{array}$ & Entrevistas semiestruturadas \\
\hline PETP & INEA & $\begin{array}{c}{ }^{\star} \text { Boscolo e Fernandes } \\
\text { (2014) } \\
\text { Artigo }\end{array}$ & comunidade rural & $\begin{array}{l}239 \text { espécies; } 12 \\
\text { categorias de uso }\end{array}$ & Entrevistas semiestruturadas \\
\hline \multirow{2}{*}{ PEIG } & \multirow{2}{*}{ INEA } & $\begin{array}{c}\text { Galvão e Esteves (2007) } \\
\text { Artigo }\end{array}$ & (a) caiçara & $\begin{array}{c}39 \text { espécies; } \\
1 \text { categoria de uso }\end{array}$ & Entrevistas semiestruturadas \\
\hline & & $\begin{array}{c}\text { Galvão (2012) } \\
\text { Dissertação ou tese }\end{array}$ & $\mathrm{s} / \mathrm{i}$ & $\begin{array}{l}162 \text { e } 172 \text { espécies; } \\
7 \text { categorias de uso }\end{array}$ & Entrevistas semiestruturadas \\
\hline \multirow{3}{*}{$\begin{array}{l}\text { REBIO } \\
\text { Poço das } \\
\text { Antas }\end{array}$} & \multirow{3}{*}{ ICMBIO } & $\begin{array}{c}\text { Christo et al. (2006) } \\
\text { Artigo }\end{array}$ & (a) sitiantes & $\begin{array}{c}210 \text { espécies; } \\
9 \text { categorias de uso }\end{array}$ & Entrevistas semiestruturadas \\
\hline & & $\begin{array}{c}\text { Christo et al. (2012) } \\
\text { Artigo }\end{array}$ & (a) sitiantes & $\begin{array}{c}129 \text { espécies; } \\
9 \text { categorias de uso }\end{array}$ & Entrevistas semiestruturadas \\
\hline & & $\begin{array}{c}\text { Guedes et al. (2011) } \\
\text { Artigo }\end{array}$ & $\begin{array}{l}\text { (a) comunidade rural, } \\
\text { mateiros }\end{array}$ & $\begin{array}{c}143 \text { espécies; } \\
3 \text { categorias de uso }\end{array}$ & Entrevistas semiestruturadas \\
\hline
\end{tabular}




\begin{tabular}{|c|c|c|c|c|c|}
\hline UC & Gestão & $\begin{array}{l}\text { Autor/Tipo de Publi- } \\
\text { cação }\end{array}$ & Grupos estudados & Resultados & Obtenção dos dados \\
\hline \multirow{3}{*}{$\begin{array}{l}\text { REBIO } \\
\text { Tinguá }\end{array}$} & \multirow{3}{*}{ ICMBIO } & $\begin{array}{c}\text { Sobrinho et al. (2011) } \\
\text { Artigo }\end{array}$ & $\begin{array}{c}\text { (a) comunidade rural, } \\
\text { mateiros }\end{array}$ & $\begin{array}{c}77 \text { espécies; } \\
1 \text { categoria de uso }\end{array}$ & Entrevistas semiestruturadas \\
\hline & & $\begin{array}{c}\text { Guedes et al. (2011) } \\
\text { Artigo }\end{array}$ & $\begin{array}{l}\text { (b) comunidade rural, } \\
\text { mateiros }\end{array}$ & $\begin{array}{c}60 \text { espécies; } \\
3 \text { categorias de uso }\end{array}$ & Entrevistas semiestruturadas \\
\hline & & $\begin{array}{c}\text { Oliveira et al. (2011) } \\
\text { Artigo }\end{array}$ & (a) sitiantes & $\begin{array}{c}72 \text { espécies; } \\
1 \text { categoria de uso }\end{array}$ & Entrevistas semiestruturadas \\
\hline \multirow{4}{*}{ REJ } & \multirow{4}{*}{ INEA } & $\begin{array}{c}\text { Borges e Peixoto (2009) } \\
\text { Artigo }\end{array}$ & (a) caiçaras & $\begin{array}{c}76 \text { espécies; } \\
5 \text { categorias de uso }\end{array}$ & Entrevistas semiestruturadas \\
\hline & & $\begin{array}{l}\text { Brito e Valle (2011) } \\
\qquad \text { Artigo }\end{array}$ & (a) caiçaras & $\begin{array}{c}89 \text { espécies; } \\
1 \text { categoria de uso }\end{array}$ & Entrevistas semiestruturadas \\
\hline & & $\begin{array}{l}\text { Brito e Valle (2012) } \\
\qquad \text { Artigo }\end{array}$ & (a) caiçaras & $\begin{array}{c}190 \text { táxons; } \\
9 \text { categorias de uso }\end{array}$ & Entrevistas semiestruturadas \\
\hline & & $\begin{array}{l}\text { Idrobo et al (2016) } \\
\qquad \text { Artigo }\end{array}$ & caiçaras & $\mathrm{s} / \mathrm{i}$ & Entrevistas semiestruturadas \\
\hline
\end{tabular}


Tabela 4. As Unidades de Conservaçăo de Uso Sustentável (federais e estaduais) no estado do Rio de Janeiro com publicaçồes em Etnobotânica. Legenda: APA (Área de Proteçấo Ambiental) - APAC (APA Cairuçu), APACG (APA da Capoeira Grande), APAP (APA Palmares) APAS (APA de Sepetiba), APAMC (APA de Macaé de Cima), APASM (APA da Serra da Mantiqueira). RESEXMAR-AC (Reserva Extrativista Marinha do Arraial do Cabo), RPPN (Reserva Particular do Patrimônio Nacional), RPPN-CEGHN (RPPN Campo Escoteiro Geraldo Hugo Nunes), RPPN-SA (RPPN Santo Antônio), RPPN-NA (RPPN da Agulhas Negras), RPPN-DP (RPPN Dois Peôes). (a) Populaçầo tradicional; (b) Populaçầo năo tradicional. (E) Entrevista pesquisa etnobotânica utilizando técnicas de entrevistas. (*) Pesquisa relevante em mais de uma UC.

\begin{tabular}{|c|c|c|c|c|c|}
\hline $\mathrm{UC}$ & Gestão & Autor & $\begin{array}{l}\text { Grupos } \\
\text { estudados }\end{array}$ & No. de espécies & Obtenção dos dados \\
\hline APAC & ICMBIO & $\begin{array}{c}\text { Borges e Peixoto (2009) } \\
\text { Artigo }\end{array}$ & (a) caiçaras & $\begin{array}{c}76 \text { espécies; } \\
5 \text { categorias de uso }\end{array}$ & Entrevistas semiestruturadas \\
\hline APACG & Municipal & $\begin{array}{c}\text { Patzlaff et al. (2011) } \\
\text { Artigo }\end{array}$ & $\begin{array}{l}\text { (b) alunos e } \\
\text { professores } \\
\text { do ensino } \\
\text { fundamental }\end{array}$ & $\begin{array}{c}70 \text { espécies; } \\
1 \text { categorias de uso }\end{array}$ & Entrevistas semiestruturadas \\
\hline APAP & INEA & $\begin{array}{l}\text { Valverde et al. (2018) } \\
\text { Artigo }\end{array}$ & $\begin{array}{l}\text { (a) comuni- } \\
\text { dade rural }\end{array}$ & $\begin{array}{c}76 \text { espécies; } \\
1 \text { categoria de uso }\end{array}$ & Entrevistas semiestruturadas \\
\hline APAS & INEA & $\begin{array}{l}\text { Figueiredo et al. (1997) } \\
\text { Artigo }\end{array}$ & (a) caiçaras & $\begin{array}{c}75 \text { espécies; } \\
3 \text { categorias de uso }\end{array}$ & Entrevistas semiestruturadas \\
\hline APAMC & INEA & $\begin{array}{c}{ }^{\star} \text { Boscolo e Fernandes } \\
\qquad(2014) \\
\text { Artigo }\end{array}$ & $\begin{array}{l}\text { (a) comuni- } \\
\text { dade rural }\end{array}$ & $\begin{array}{c}239 \text { espécies: } \\
12 \text { categorias de uso }\end{array}$ & Entrevistas semiestruturadas \\
\hline
\end{tabular}




\begin{tabular}{|c|c|c|c|c|c|}
\hline UC & Gestão & Autor & $\begin{array}{l}\text { Grupos } \\
\text { estudados }\end{array}$ & No. de espécies & Obtenção dos dados \\
\hline \multirow{2}{*}{ APASM } & \multirow{2}{*}{ ICMBIO } & $\begin{array}{c}\text { ^Baldini (2015) } \\
\text { Dissertação ou tese }\end{array}$ & $\begin{array}{l}\text { (a) sitiantes, } \\
\text { comunidade } \\
\text { rural }\end{array}$ & $\begin{array}{l}294 \text { espécies: } \\
6 \text { categorias de uso }\end{array}$ & Entrevistas semiestruturadas \\
\hline & & $\begin{array}{l}\text { Quinteiro et al. (2015) } \\
\text { Artigo }\end{array}$ & $\begin{array}{l}\text { (a)comunida- } \\
\text { de rural }\end{array}$ & $\begin{array}{c}27 \text { espécies; } \\
4 \text { categorias de uso }\end{array}$ & Entrevistas semiestruturadas \\
\hline $\begin{array}{l}\text { RESEXMAR- } \\
-A C\end{array}$ & ICMBIO & $\begin{array}{l}\text { Fonseca-Kruel e Peixoto } \\
\qquad \begin{array}{c}\text { (2004) } \\
\text { Artigo }\end{array}\end{array}$ & $\begin{array}{l}\text { (a) pescado- } \\
\text { res artesanais }\end{array}$ & $\begin{array}{l}68 \text { espécies; } \\
6 \text { categorias de uso }\end{array}$ & Entrevistas semiestruturadas \\
\hline $\begin{array}{l}\text { RPPN-CE- } \\
\text { GHN }\end{array}$ & Particular & $\begin{array}{l}\text { Mourão et al. (2017) } \\
\text { Artigo }\end{array}$ & (a) sitiantes & $\begin{array}{l}76 \text { espécies; } 6 \text { cate- } \\
\text { gorias de uso; (E) }\end{array}$ & Entrevistas semiestruturadas \\
\hline RPPN-SA & \multirow{3}{*}{ Particular } & \multirow{3}{*}{$\begin{array}{c}\text { ^Baldini (2015) } \\
\text { Dissertação ou tese }\end{array}$} & \multirow{3}{*}{$\begin{array}{l}\text { (a) sitiantes, } \\
\text { comunidade } \\
\text { rural }\end{array}$} & \multirow{3}{*}{$\begin{array}{l}294 \text { espécies; } \\
6 \text { tipos de uso }\end{array}$} & \multirow{3}{*}{ Entrevistas semiestruturadas } \\
\hline RPPN-NA & & & & & \\
\hline RPPN-DP & & & & & \\
\hline
\end{tabular}




\section{Consideraçồes finais}

Cresce, no Brasil, o número de áreas protegidas e das pesquisas científicas nessas áreas, mas, dado o tamanho do país, da diversidade de biomas e da biodiversidade embutida, é preciso mudar o paradigma das atividades científicas nesses espaços tão necessários nos dias atuais. As comparações e análises aqui realizadas permitiram identificar lacunas tanto nos conhecimentos acadêmicos como em ações para conservação da biodiversidade no estado do Rio de Janeiro. Há demanda de estudos sobre o conhecimento tradicional e local associado à biodiversidade fluminense para que estes integrem o processo de gestão das UCs. Com isso, o conhecimento tradicional pode auxiliar na elaboração de regras (identificando zonas de recursos vegetais) e no plano de manejo das UCs, em ações de educação ambiental e intensificação de medidades de proteção ambiental.

As pesquisas etnobotânicas em unidades de conservação fluminenses poderiam ser prioritariamente dirigidas para as UCs, onde as finalidades sejam mais afeitas ao uso dos recursos, como as UCs de Uso Sustentável. Entretanto, isso não significa que as UCs de Proteção Integral não sejam estudadas sob esse aspecto, já que tanto as UCs de Proteção Integral quanto as de desenvolvimento sustentável do território fluminense apresentam em seu histórico algum uso pretérito de recursos pelas populações tradicionais. Além disso, a ocupação desses territórios precede em muito o estabelecimento de unidades de conservação no Brasil. Independente da categoria de UC a ser investigada, é recomendável que o pesquisador não se comporte como simples usuário, ${ }^{16}$ mas que, na medida do possível, sua pesquisa se traduza em ações para a proteção, gestão e conservação da UC. Para tanto, o plano de manejo da UC (quando houver) é leitura fundamental na formulação da pesquisa.

${ }^{16}$ Luz e Elias, 2014. 


\section{Referências}

ALBUQUERQUE, U. P. Introdução à etnobotânica. 2 ed. Rio de Janeiro: Interciência, 2005.

ALCORN, J. B. "The scope and aims of ethnobotany in a developing world”. In SCHULTES, R. E. e REIS S. V. (eds.). Ethnobotany: evolution of a discipline. Cambridge: Timber Press, 1995, pp. 23-39.

ALEXIADES, M. N. e SHELDON, J. W. (eds.). Ethnobotanical research: a field manual. Nova Iorque: The New York Botanical Garden, 1996.

ALVES, R. P. e HANAZAKI, N. "Áreas protegidas marinho-costeiras de Santa Catarina sob a perspectiva das populações locais: contribuições da literatura”. Ambiente \& Sociedade, v. 18, n. 4, pp. 97-118, 2015.

BALDINI, K. B. L. Relação homem-natureza em grupos rurais (caipiras, sitiantes e pequenos agricultores) na Mata Atlântica e conservação da biodiversidade em um município do Sul Fluminense, $R J$ (tese). Universidade Federal Rural do Rio de Janeiro, 2015.

e SILVA, J. G. "Madeiras do Parque Nacional do Itatiaia: etnobotânica e conservação dos recursos naturais". Floresta e Ambiente, v. 14, n. 2, pp. 35-48, 2007.

BARROS, W. D. Parques Nacionais do Brasil, Série Documentária n. 1, Rio de Janeiro, Ministério da Agricultura, 1952.

BAUMGRATZ, J. F. A et al. Catálogo das espécies de plantas vasculares e briófitas do estado do Rio de Janeiro. Instituto de Pesquisas Jardim Botânico do Rio de Janeiro, 2014. Disponível em: http://florariojaneiro.jbrj.gov. br/. Acesso em: 20 fev. 2015.

BERKES, F. et al. "Traditional ecological knowledge, biodiversity, resilience and sustainability". In PERRINGS C. S. et al. (eds.). Biodiversity conservation: problems and policies. Dordrecht: Kluwer Academic Press, 1995, pp. 281-300.

BITTENCOURT, L. A. F. e PAULA, A. "Análise cienciométrica de produção científica em unidades de conservação federais no Brasil”. Enciclopédia Biosfera, v. 8, n. 14, pp. 2044-54, Goiânia, 2012. 
BORGES, R. e PEIXOTO, A. L. "Conhecimento e uso de plantas em uma comunidade caiçara do litoral sul do estado do Rio de Janeiro, Brasil". Acta Botanica Brasílica, v. 23, n. 3, pp. 769-79, 2009.

BOSCOLO, O. H. Estudos etnobotânicos nas comunidades de Galdinópolis e Rio Bonito, Nova Friburgo, RJ (tese). UFRJ, 2011.

e FERNANDES, L. "Appropriation of biodiversity and traditional knowledge: a case study of two rural communities in Rio de Janeiro state, Brazil”. Scientia Plena, v. 10, n. 11, nov. 2014.

e SENNA-VALLE, L. "Plantas de uso medicinal em Quissamã, Rio de Janeiro, Brasil”. Iheringia, Série Botânica, v. 63, pp. 263-77, 2008.

BRASIL. "Lei n. ${ }^{\circ}$ 9.985, de 18 de julho de 2000”. Regulamenta o artigo 225, $\S 1^{\circ}$,incisos I, II, III e VII da Constituição Federal, institui o Sistema Nacional de Unidades de Conservação da Natureza e dá outras providências. Ministério do Meio Ambiente. Brasília, 2000. Disponível em: http://www.planalto.gov.br/ccivil_03/Leis/L9985.htm. Acesso em: 27 maio 2018.

BRITO, M. R. de e SENNA-VALLE, L. "Plantas medicinais utilizadas na comunidade caiçara da Praia do Sono, Paraty, Rio de Janeiro, Brasil”. Acta Botanica Brasílica, v. 25, n. 2, pp. 363-72, 2011.

e . "Diversity of plant knowledge in a caiçara community from the Brazilian Atlantic Forest coast". Acta Botanica Brasílica, v. 26, n. 4, pp. 375-747, Feira de Santana, out.-dez. 2012.

CHRISTO, A. G. et al. "Uso de recursos vegetais em comunidades rurais limítrofes à reserva biológica de Poço das Antas, Silva Jardim, Rio de Janeiro: estudo de caso na gleba Aldeia Velha”. Rodriguésia, v. 57, pp. 519-42, Rio de Janeiro, 2006.

et al. "Evidence for conservation and sustainable use in a fragment of the Atlantic Forest in southeastern Brazil by a traditional human group”. SpringerPlus, v. 1, pp. 1-21, 2012.

CUNNINGHAM, A. Applied ethnobotany: people, wild plant use and conservation. London: Earthscan, 2000. 
FIGUEIREDO, G. M. et al. "Ethnobotany of Atlantic Forest coastal communities: II. Diversity of plant uses at Sepetiba bay (SE Brazil)". Human Ecology, v. 25, pp. 353-60, 1997.

FONSECA-KRUEL, V. S. Etnobotânica nas restingas no Centro de Diversidade Vegetal de Cabo Frio, Rio de Janeiro, Brasil (tese). Escola Nacional de Botânica Tropical, 2011.

e PEIXOTO, A. L. "Etnobotânica na Reserva Extrativista Marinha de Arraial do Cabo, RJ, Brasil”. Acta Botanica Brasílica, v. 18, pp. 177-90, 2004.

et al. "Quantitative ethnobotany of a restinga forest fragment in Rio de Janeiro, Brasil”. Rodriguésia, v. 60, pp. 137-41, 2009.

FORD, R. I. "Ethnobotany: historical diversity and synthesis". In et

al. (eds.). The nature and status of ethnobotany: Annals of Arnold Arboretum - Anthropological Papers, v. 67, pp. 33-49, Michigan, 1978.

GALVÃO, M. N. e ESTEVES, R. L. "Plantas medicinais utilizadas pela comunidade de Vila Dois Rios, Ilha Grande, Rio de Janeiro, Brasil”. Bradea, v. 12, p. 10, 2007.

. Etnobotânica na Ilha Grande, Angra dos Reis, Rio de Janeiro: uso de recursos vegetais na Vila do Abraão e Praia Longa (tese). UFRJ, 2012.

GUEDES-BRUNI, R. R. et al. "Mateiros, matas e reservas: saber local e conservação". In PEIXOTO, A. L. e SILVA, I. M. (orgs.). Saberes e usos de plantas: legados de atividades humanas no Rio de Janeiro. $1 \mathrm{ed}$. Rio de Janeiro: Editora PUC-Rio, 2011, pp. 93-128.

HANAZAKI, N. et al. "Diversity of plant uses in two caiçara communities from the Atlantic Forest coast, Brazil". Biodiversity and Conservation, v. 9, pp. 597-615, 2000.

ICMBIO - Instituto Chico Mendes de Conservação da Biodiversidade. Disponível em: http://www.brasil.gov.br/editoria/turismo/2018/02/ parques-nacionais-tem-recorde-de-visitacao-conheca-algumas-das-atracoes. Acessado em: 22 maio 2018.

IDROBO, C. J. et al. "Produced natures through the lens of biodiversity conservation and tourism: the Ponta Negra caiçara in the Atlantic Forest coast of Brazil”. Local Environment, v. 21, n. 9, pp. 1132-50, 2016. 
INEA - Instituto Estadual do Ambiente. Disponível em: http://www. inea.rj.gov.br/Portal/Agendas/BIODIVERSIDADEEAREASPROTEGIDAS/UnidadesdeConservacao/index.htm. Acesso em: 22 maio 2018.

Secretaria do ambiente. RPPN reconhecidas pelo Instituto Estadual do Ambiente. Disponível em: http://www.inea.rj.gov.br/cs/groups/ public/documents/document/zwew/mtuz/ edisp/inea0153384.pdf. Acesso em: 22 maio 2018.

LOPES. R. C. e ANDREATA, R. H. P. "Plantas medicinais do Pico Alto Mourão I”. Eugeniana, v. 16, pp. 1-9, Rio de Janeiro, 1989.

. "Plantas medicinais do Pico Alto Mourão II”. Eugeniana, v. 17, pp. 15-21, Rio de Janeiro, 1990.

. "Plantas Medicinais do Pico Alto Mourão III”. Eugeniana, v. 18, pp. 21-30, Rio de Janeiro, 1991.

. "Plantas Medicinais do Pico Alto Mourão IV". Eugeniana, v. 19, pp. 1-10, Rio de Janeiro, 1992.

et al. "Plantas ornamentais ocorrentes na Mata Atlântica do morro Alto Mourão, Niterói/Maricá, Rio de Janeiro”, Publicações Avulsas do Museu Nacional, v. 102, pp. 3-14, Rio de Janeiro, 2004.

LUIJK N. V. e FONSECA-KRUEL, V.S. Transmissão do conhecimento etnobotânico sobre as plantas úteis da restinga de Arraial do Cabo, RJ (monografia). Instituto Federal Fluminense, 2017.

LUZ, A. P. e ELIAS, H. T. "Pesquisa científica em unidades de conservação”. Revista Agropecuária Catarinense, v. 27, n. 1, pp. 21-4, 2014.

MAGALHÃES, A. C. Etnobotânica, saberes locais e agricultura no contex to de uma floresta urbana: Maciço da Pedra Branca, RJ (dissertação). Pontifícia Universidade Católica do Rio de Janeiro, 2010.

MAGNANINI, C. Etnobotânica em Itatiaia, Rio de Janeiro: plantas medicinais do Parque Nacional do Itatiaia e áreas do entorno (dissertação). Universidade Federal Fluminense, 2005.

MAIOLI-AZEVEDO, V. e SENNA-VALLE, L. "Quilombolas da Fazenda Machadinha: tradição e uso dos recursos vegetais”. In PEIXOTO, A. 
L. e SILVA, I. M. (orgs.). Saberes e usos de plantas: legados de atividades humanas no Rio de Janeiro, v. 1, pp. 129-72, Rio de Janeiro, 2011.

MEDEIROS, M. F. T. et al. "Plantas medicinais e seus usos pelos sitiantes da Reserva Rio das Pedras, Mangaratiba, RJ, Brasil”. Acta Botanica Brasílica, v. 18, n. 2, pp. 391-9, 2004.

et al. Flora medicinal dos sitiantes da Reserva Particular do Patrimônio Natural do Rio das Pedras, Mangaratiba, Rio de Janeiro, Brasil: taxonomia e aspectos etnobotânicos”,. Publicações Avulsas do Museu Nacional, v. 106, pp. 1-24, Rio de Janeiro, 2005.

MMA - Ministério do Meio Ambiente. Cadastro Nacional de Unidades de Conservação. Disponível em: http://www.mma.gov.br/areas-protegidas/cadastro-nacional-de-ucs. Acesso em: 22 maio 2018.

MOURÃO, M. D. S. et al. "Conhecimento local e uso dos recursos vegetais na comunidade Sertão, Magé, Rio de Janeiro”. Revista Fitos Eletrônica, pp. 1-126, 2017.

OLIVEIRA, L. S. et al. "Plantas medicinais como recurso terapêutico em comunidade do entorno da Reserva Biológica do Tinguá, RJ, Brasil: metabólitos secundários e aspectos farmacológicos". InterSciencePlace, v. 1, n. 17, 2015.

PATZLAFF, R. G. et al. "O saber local sobre plantas de uso medicinal em uma escola pública no entorno de um fragmento de floresta atlântica, em Guaratiba, Rio de Janeiro, RJ”. In PEIXOTO, A. L. e SILVA, I. M. (orgs.). Saberes e usos de plantas: legados de atividades humanas no Rio de Janeiro. 1 ed. Rio de Janeiro: Editora PUC-Rio, 2011, pp. 173-224.

QUINTEIRO, M. M. C. et al. "Inventory and implications of plant use for environmental conservation in Visconde de Maua, Serra da Mantiqueira, Brazil”. Ethnobotany Research and Applications, v. 14, pp. 27-47, 2015.

ROCHA, J. A. et al. "Etnobotânica: um instrumento para valorização e identificação de potenciais de proteção do conhecimento tradicional”. Interações, v. 16, n. 1, pp. 67-74, 2015.

RYLANDS, A. B. e BRANDON, K. "Unidades de conservação brasileiras". Megadiversidade, v. 1, n. 1, pp. 27-35, 2005. 
SANTA CATARINA. "Lei n. ${ }^{\circ} 11.986$, de 12 de novembro de 2001". Institui o Sistema Estadual de Unidades de Conservação da Natureza e adota outras providências. Florianópolis, 2001.

SANTILLI, J. "Áreas protegidas e direitos de povos e comunidades tradicionais". In BENSUSAN N. e PRATES A. (orgs.). A diversidade cabe na unidade?. Brasília: IEB, 2014, pp. 470-507.

SANTOS, M. G. et al. "Recursos vegetais da Restinga de Carapebus, Rio de Janeiro, Brasil”. Revista de Biologia Neotropical, v. 6, n. 1, pp. 35-54, 2009.

SCHULTES, R. E. e REIS, S. V. (eds.). Ethnobotany: evolution of a discipline. Cambridge: Timber Press, 1995.

SOBRINHO, F. D. A. P. et al. "Uso de plantas medicinais no entorno da Reserva Biológica de Tinguá, Nova Iguaçu, RJ”. Revista Acadêmica: $\mathrm{Ci}$ ência Animal, v. 9, n. 2, pp. 195-206, 2011.

VALVERDE, A. V. et al. "Introdução da Fitoterapia no SUS: contribuindo com a Estratégia de Saúde da Família na comunidade rural de Palmares, Paty do Alferes, Rio de Janeiro". Revista Fitos Eletrônica, v. 12, n. 1, pp. 27-40, 2018. 